parison of the limits given by (2) and by Tchebycheff's theorem, Fig. 2 has been prepared. This shows the values of $1-P_{t \sigma}$ plotted against $t$, the several curves corresponding to various values of $\rho$ as indicated. The dotted line gives the limits from Tchebycheff's theorem.

Bell Telephone Laboratories

\title{
CHARACTERISTICS OF MULTIPLE CURVES AND THEIR RESIDUALS*
}

\section{BY T. R. HOLLCROFT}

Salmon $\dagger$ obtained formulas relating the characteristics of two curves which together form the complete intersection of two algebraic surfaces when one of the curves is double on one of the surfaces. In this paper, by a generalization of Salmon's method, the relations between the characteristics of two such curves are found when one of the curves is of given multiplicity on each of the two surfaces. Such a formula is useful in studying a system. of surfaces with a multiple basis curve. It was this need for it that led to its derivation.

Consider two algebraic surfaces $f_{1}$ and $f_{2}$ of orders $\mu_{1}$ and $\mu_{2}$, respectively, whose complete intersection consists of two curves $C_{1}, C_{2}$ of orders $n_{1}, n_{2} ;$ ranks $r_{1}, r_{2}$; genera $p_{1}, p_{2}$; and with $h_{1}, h_{2}$ apparent double points, respectively. Assume that $C_{1}$ is of multiplicity $i_{1}$ on $f_{1}$ and $i_{2}$ on $f_{2}$ and also that $C_{1}$ itself is the complete intersection curve of two surfaces. $C_{1}$ (counted simply) and $C_{2}$ have $t$ actual intersections and $n_{1} n_{2}-t$ apparent intersections.

Consider a third surface $f_{3}$ of order $\mu_{3}$ passing simply through $C_{1}$ but not through $C_{2}$. The equivalence $E$ of $C_{1}$ on the three surfaces $f_{1}, f_{2}, f_{3}$ is $\ddagger$

$$
E=n_{1}\left(i_{2} \mu_{1}+i_{1} \mu_{2}+i_{1} i_{2} \mu_{3}-2 i_{1} i_{2}\right)-i_{1} i_{2} r_{1} .
$$

*Presented to the Society, April 14, 1933.

$\dagger$ Salmon, Geometry of Three Dimensions, 4th ed., 1882, p. 322.

$\ddagger$ M. Noether, Sulle curve multiple di superficie algebriche, Annali di Matematica, (2), vol. 5 (1871), p. 166. 
The number of points of intersection of $f_{1}, f_{2}, f_{3}$ outside of $C_{1}$ is, therefore, $\mu_{1} \mu_{2} \mu_{3}-E$.

The surfaces $f_{1}$ and $f_{2}$ intersect in the curve $C_{1}$ counted $i_{1} i_{2}$ times and the residual curve $C_{2}$ of order $n_{2}=\mu_{1} \mu_{2}-i_{1} i_{2} n_{1}$. Since $f_{3}$ does not pass through $C_{2}$, the number of intersections of $f_{1}, f_{2}, f_{3}$ outside of $C_{1}$ equals the number of intersections of $C_{2}$ and $f_{3}$ less the number $t$ of actual intersections of $C_{1}$ and $C_{2}$. Equating the two expressions for the number of intersections of $f_{1}, f_{2}, f_{3}$ outside of $C_{1}$, we have

$$
\mu_{3}\left(\mu_{1} \mu_{2}-i_{1} i_{2} n_{1}\right)-t=\mu_{1} \mu_{2} \mu_{3}-E,
$$

and solving for $t$, we find

$$
t=n_{1}\left(i_{2} \mu_{1}+i_{1} \mu_{2}-2 i_{1} i_{2}\right)-i_{1} i_{2} r_{1} .
$$

In order to find an expression for $t$ in terms of the characteristics of $C_{2}$, consider the locus $F$ of points whose polar planes with respect to $f_{1}$ and $f_{2}$ meet an arbitrary line at one point. This surface $F$ is of order $\mu_{1}+\mu_{2}-2$. $^{*}$ Since the curve $C_{1}$ is $i_{1}$-fold on $f_{1}$ and $i_{2}$-fold on $f_{2}$, it is of multiplicity $i_{1}+i_{2}-2$ on $F$.

The residual curve $C_{2}$ meets $F$ in $n_{2}\left(\mu_{1}+\mu_{2}-2\right)$ points. Since $F$ contains $C_{1}$ as an $\left(i_{1}+i_{2}-2\right)$-fold curve and since the surfaces $f_{1}$ and $f_{2}$ have contact at each of the $t$ actual intersections of $C_{1}$ and $C_{2}$, these intersections count as $\left(i_{1}+i_{2}-1\right) t$ intersections of $C_{2}$ and $F$. Also, $C_{2}$ and $F$ intersect in the $r_{2}$ points of contact of the $r_{2}$ tangents to $C_{2}$ which meet the arbitrary line associated with $F$. Equating the two expressions for the number of intersections of $C_{2}$ and $F$, we find

$$
\left(i_{1}+i_{2}-1\right) t+r_{2}=n_{2}\left(\mu_{1}+\mu_{2}-2\right) .
$$

Eliminating $t$ from (A) and (B) and simplifying, we obtain

$$
\begin{aligned}
r_{2}-i_{1} i_{2}\left(i_{1}+i_{2}-1\right) r_{1} & =n_{2}\left(\mu_{1}+\mu_{2}-2\right) \\
& -n_{1}\left(i_{1}+i_{2}-1\right)\left(i_{2} \mu_{1}+i_{1} \mu_{2}-2 i_{1} i_{2}\right) .
\end{aligned}
$$

Substituting $r_{k}=n_{k}\left(n_{k}-1\right)-2 h_{k},(k=1,2)$, in (1), we find the following relation involving apparent double points:

$$
\begin{aligned}
& 2 h_{2}-2 i_{1} i_{2}\left(i_{1}+i_{2}-1\right) h_{1}=n_{1}\left(i_{1}+i_{2}-1\right)\left[i_{2} \mu_{1}+i_{1} \mu_{2}\right. \\
&-\left.i_{1} i_{2}\left(n_{1}+1\right)\right]-n_{2}\left(\mu_{1}+\mu_{2}-n_{2}-1\right) .
\end{aligned}
$$

* Salmon, loc. cit., pp. 308-309. 
Substituting $2 h_{k}=\left(n_{k}-1\right)\left(n_{k}-2\right)-2 p_{k},(k=1,2)$, in (2), we obtain the following relation involving the genus:

$$
\begin{aligned}
2\left(p_{2}-1\right)-2 i_{1} i_{2}\left(i_{1}+\right. & \left.i_{2}-1\right)\left(p_{1}-1\right)=n_{2}\left(\mu_{1}+\mu_{2}-4\right) \\
& -n_{1}\left(i_{1}+i_{2}-1\right)\left(i_{2} \mu_{1}+i_{1} \mu_{2}-4 i_{1} i_{2}\right) .
\end{aligned}
$$

Formulas (1), (2), (3) define the characteristics of either curve when those of the other and the multiplicities of $C_{1}$ are known. The order and multiplicities of $C_{1}$ must satisfy the inequality

$$
n_{1} i_{k}\left(i_{k}-1\right) \leqq\left(\mu_{k}-1\right)\left(\mu_{k}-2\right), \quad(k=1,2) .^{*}
$$

When the multiple curve $C_{1}$ is the complete intersection of $f_{1}$ and $f_{2}$, the equation $\mu_{1} \mu_{2}=i_{1} i_{2} n$ and the above formulas become (on setting $n_{2}=r_{2}=0$ and dropping the subscripts of the characteristics of $C_{1}$ ):

$$
\begin{aligned}
i_{1} i_{2} r & =n\left(i_{2} \mu_{1}+i_{1} \mu_{2}-2 i_{1} i_{2}\right), \\
2 i_{1} i_{2} h & =n\left(\mu_{1}-i_{1}\right)\left(\mu_{2}-i_{2}\right), \\
2 i_{1} i_{2}(p-1) & =n\left(i_{2} \mu_{1}+i_{1} \mu_{2}-4 i_{1} i_{2}\right) .
\end{aligned}
$$

The above methods and formulas are valid for all values of order and multiplicity satisfying the necessary conditions, provided that $C_{1}$ counted $i_{1} i_{2}$ times and $C_{2}$ together constitute the complete intersection of $f_{1}$ and $f_{2}$ and provided further that $C_{1}$ is itself a complete intersection of two surfaces.

The assumption that $C_{1}$ is the complete intersection of two surfaces is necessary because the equivalence formula used for $C_{1}$ was derived by Noether $\dagger$ only for a complete intersection curve. However, it has been stated by Hudson, $\ddagger$ on evidence not sufficient for proof, that this equivalence formula is very probably valid for all space curves of given order and rank whether complete intersections or not. It is, therefore, probable that the formulas derived above hold for all space curves, but no proof of this is available.

Wells College

* For $i_{k}>2$ and large values of $\mu_{k}$, this inequality must be replaced by another. See T. R. Hollcroft, Multiple points of algebraic curves, this Bulletin, vol. 35 (1929), pp. 848-849.

$\dagger$ M. Noether, loc. cit.

$\ddagger$ H. Hudson, Cremona Transformations, 1927, p. 221, end of $\$ 15$. 